\title{
SIMULTANEOUS EXACT CONTROLLABILITY AND SOME APPLICATIONS*
}

\author{
MARIUS TUCSNAK ${ }^{\dagger}$ AND GEORGE WEISS T $^{\ddagger}$
}

\begin{abstract}
We study the exact controllability of two systems by means of a common finitedimensional input function, a property called simultaneous exact controllability. Most of the time we consider one system to be infinite-dimensional and the other finite-dimensional. In this case we show that if both systems are exactly controllable in time $T_{0}$ and the generators have no common eigenvalues, then they are simultaneously exactly controllable in any time $T>T_{0}$. Moreover, we show that similar results hold for approximate controllability. For exactly controllable systems we characterize the reachable subspaces corresponding to input functions of class $H^{1}$ and $H^{2}$. We apply our results to prove the exact controllability of a coupled system composed of a string with a mass at one end. Finally, we consider an example of two infinite-dimensional systems: we characterize the simultaneously reachable subspace for two strings controlled from a common end. The result is obtained using a recent generalization of a classical inequality of Ingham.
\end{abstract}

Key words. linear system, operator semigroup, admissible control operator, Gramian, exact controllability, exact observability, simultaneous controllability, wave equation, boundary control, coupled system

AMS subject classifications. 93B28, 93C25, 93B03, 93C20

PII. S0363012999352716

1. Introduction. We consider two control systems (possibly infinite-dimensional), with the states denoted by $z_{1}, z_{2}$, described by the equations

$$
\begin{cases}\dot{z}_{1}(t)=A_{1} z_{1}(t)+B_{1} u(t), & z_{1}(0)=0 \\ \dot{z}_{2}(t)=A_{2} z_{2}(t)+B_{2} u(t), & z_{2}(0)=0 .\end{cases}
$$

Here, a dot denotes differentiation with respect to the time $t, A_{1}, A_{2}$ are generators of strongly continuous operator semigroups on the corresponding state spaces, and $B_{1}, B_{2}$ are admissible control operators for these semigroups. Note that the two systems receive the same input function $u$. These systems are called simultaneously exactly controllable in time $T$ (where $T>0$ ), if for any states $f_{1}$ and $f_{2}$, an $L^{2}$-function $u$ can be found such that $z_{1}(T)=f_{1}$ and $z_{2}(T)=f_{2}$.

Simultaneous exact controllability was first considered by Russell in [22] and it is the subject of Chapter 5 in Lions [20]. The simultaneous controllability of two Riesz spectral systems (one hyperbolic and one parabolic) was studied in section 4 of Hansen [10] (see also Hansen and Zhang [12]). We were led to investigate simultaneous exact controllability in our study of coupled systems (sometimes called hybrid systems), such as a string with a mass at one end, or the SCOLE model of a beam clamped at one end and with a rigid body at the other end.

Our main result (proved in section 3) concerns the situation where one system is finite-dimensional. We show that, in this case, if $A_{1}$ and $A_{2}$ have no common eigenvalues and if both are exactly controllable in time $T_{0}$, then they are simultaneously

* Received by the editors March 1, 1999; accepted for publication (in revised form) October 4, 1999; published electronically May 11, 2000.

http://www.siam.org/journals/sicon/38-5/35271.html

${ }^{\dagger}$ Department of Mathematics, University of Nancy-I, POB 239, Vandoeuvre les Nancy 54506, France (marius.tucsnak@iecn.u-nancy.fr).

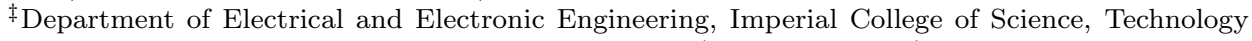
and Medicine, Exhibition Road, London SW7 2BT, UK (g.weiss@ic.ac.uk). 
exactly controllable in any time $T>T_{0}$. For $T=T_{0}$ this is not always true, as we show in an example (see section 4 ).

The concept of simultaneous approximate controllability of two systems in time $T$ is similar to the controllability concept defined earlier, but now the reachable pairs of states $\left(f_{1}, f_{2}\right)$ must be dense in the product of the respective state spaces. Considering again one system to be finite-dimensional, we have a result that resembles our main result, but now we have no information on the time $T$ needed for simultaneous approximate controllability: we only know that some $T>0$ will work. Other results in section 3 concern the characterization of the reachable subspace of an exactly controllable system, when the input function $u$ is constrained to be in the Sobolev space $H^{1}$ (or $H^{2}$ ) with $u(0)=0$ (or with $u(0)=\dot{u}(0)=0$ ).

In section 4 we give two applications to systems governed by partial differential equations (PDEs), both based on the (nonhomogeneous) one-dimensional wave equation. These two interdependent examples illustrate how simultaneous controllability results can be applied in the analysis of coupled systems. In section 5 we characterize the simultaneously reachable subspace of two systems describing vibrating strings. The results here are based on recent generalizations of an inequality of Ingham.

2. Some background on infinite-dimensional systems. In this section we gather, for easy reference, some basic facts about admissible control and observation operators, controllability, and observability. Some results here are new, but most are well known. For the latter, we do not give proofs; we only refer to the relevant literature.

We assume that $X$ is a Hilbert space and $A: \mathcal{D}(A) \rightarrow X$ is the generator of a strongly continuous semigroup $\mathbb{T}$ on $X$. We define the Hilbert space $X_{1}$ as $\mathcal{D}(A)$ with the norm $\|z\|_{1}=\|(\beta I-A) z\|$, where $\beta \in \rho(A)$ is fixed (this norm is equivalent to the graph norm). The Hilbert space $X_{-1}$ is the completion of $X$ with respect to the norm $\|z\|_{-1}=\left\|(\beta I-A)^{-1} z\right\|$. This space is isomorphic to $\mathcal{D}\left(A^{*}\right)^{*}$, and we have

$$
X_{1} \subset X \subset X_{-1},
$$

densely and with continuous embeddings. $\mathbb{T}$ extends to a semigroup on $X_{-1}$, denoted by the same symbol. The generator of this extended semigroup is an extension of $A$, whose domain is $X$, so that $A: X \rightarrow X_{-1}$.

We assume that $U$ is a Hilbert space and $B \in \mathcal{L}\left(U, X_{-1}\right)$ is an admissible control operator for $\mathbb{T}$, defined as in Weiss [24]. This means that if $z$ is the solution of

$$
\dot{z}(t)=A z(t)+B u(t)
$$

(an equation in $X_{-1}$ ), with $z(0)=z_{0} \in X$ and $u \in L^{2}([0, \infty), U)$, then $z(t) \in X$ $\forall t \geq 0$. In this case, $z$ is a continuous $X$-valued function of $t$. We have

$$
z(t)=\mathbb{T}_{t} z_{0}+\Phi_{t} u,
$$

where $\Phi_{t} \in \mathcal{L}\left(L^{2}([0, \infty), U), X\right)$ is defined by

$$
\Phi_{t} u=\int_{0}^{t} \mathbb{T}_{t-\sigma} B u(\sigma) d \sigma .
$$

The above integration is done in $X_{-1}$, but the result is in $X$. The Laplace transform of $z$ is

$$
\hat{z}(s)=(s I-A)^{-1}\left[z_{0}+B \hat{u}(s)\right] .
$$


$B$ is called bounded if $B \in \mathcal{L}(U, X)$ (and unbounded otherwise).

We assume that $Y$ is another Hilbert space and $C \in \mathcal{L}\left(X_{1}, Y\right)$ is an admissible observation operator for $\mathbb{T}$, defined as in Weiss [25]. This means that for every $T>0$ there exists a $K_{T} \geq 0$ such that

$$
\int_{0}^{T}\left\|C \mathbb{T}_{t} z_{0}\right\|^{2} d t \leq K_{T}^{2}\left\|z_{0}\right\|^{2} \quad \forall z_{0} \in \mathcal{D}(A) .
$$

$C$ is called bounded if it can be extended such that $C \in \mathcal{L}(X, Y)$.

We regard $L_{l o c}^{2}([0, \infty), Y)$ as a Fréchet space with the seminorms being the $L^{2}$ norms on the intervals $[0, n], n \in \mathbb{N}$. Then the admissibility of $C$ means that there is a continuous operator $\Psi: X \rightarrow L_{l o c}^{2}([0, \infty), Y)$ such that

$$
\left(\Psi z_{0}\right)(t)=C \mathbb{T}_{t} z_{0} \quad \forall z_{0} \in \mathcal{D}(A) .
$$

The operator $\Psi$ is completely determined by $(2.6)$, because $\mathcal{D}(A)$ is dense in $X$. We introduce the $\Lambda$-extension of $C$, denoted $C_{\Lambda}$, by

$$
C_{\Lambda} z_{0}=\lim _{\lambda \rightarrow+\infty} C \lambda(\lambda I-A)^{-1} z_{0},
$$

whose domain $\mathcal{D}\left(C_{\Lambda}\right)$ consists of all $z_{0} \in X$ for which the limit exists. If we replace $C$ by $C_{\Lambda}$, formula (2.6) becomes true $\forall z_{0} \in X$ and for almost every $t \geq 0$. For $z_{0} \in \mathcal{D}(A), \Psi z_{0}$ is almost everywhere (a.e.) differentiable and

$$
\frac{d}{d t}\left(C \mathbb{T}_{t} z_{0}\right)=C_{\Lambda} \mathbb{T}_{t} A z_{0} \quad \text { for almost every } t \geq 0 .
$$

If $y=\Psi z_{0}$, then its Laplace transform is

$$
\hat{y}(s)=C(s I-A)^{-1} z_{0} .
$$

If $\mathbb{T}$ is exponentially stable, then $\Psi \in \mathcal{L}\left(X, L^{2}([0, \infty), Y)\right)$.

The following duality result holds: if $\mathbb{T}$ is a semigroup on $X$ with generator $A$, then $B \in \mathcal{L}\left(U, X_{-1}\right)$ is an admissible control operator for $\mathbb{T}$ if and only if $B^{*}: \mathcal{D}\left(A^{*}\right) \rightarrow U$ is an admissible observation operator for the dual semigroup $\mathbb{T}^{*}$. Moreover, the adjoint of $\Phi_{T}$ from (2.4) is given by

$$
\left(\Phi_{T}^{*} z_{0}\right)(t)=B_{\Lambda}^{*} \mathbb{T}_{T-t}^{*} z_{0}
$$

for almost every $t \in[0, T]$, where $B_{\Lambda}^{*} z=\lim _{\lambda \rightarrow+\infty} \lambda B^{*}\left(\lambda I-A^{*}\right)^{-1} z$, as in (2.7). For all the facts listed so far in this section, we refer to [24], [25], and [26].

For $C, \mathbb{T}$ as in (2.5) and for every $T>0$, we introduce the bounded operator $\Psi_{T}: X \rightarrow L^{2}([0, T], Y)$ by truncating $\Psi$ to $[0, T]$, i.e., $\forall t \in[0, T]$,

$$
\left(\Psi_{T} z_{0}\right)(t)=C \mathbb{T}_{t} z_{0} \quad \forall z_{0} \in \mathcal{D}(A) .
$$

The observability Gramians of $(A, C)$ are the operators

$$
P_{T}=\Psi_{T}^{*} \Psi_{T} \quad \forall T \geq 0 .
$$

Thus, for $z_{0} \in \mathcal{D}(A)$,

$$
P_{T} z_{0}=\int_{0}^{T} \mathbb{T}_{t}^{*} C^{*} C \mathbb{T}_{t} z_{0} d t
$$


and, to get an expression valid $\forall z_{o} \in X$, we may replace $C$ by $C_{\Lambda}$ in the above formula. If $\mathbb{T}$ is exponentially stable, then we may also take $T=\infty$, defining the Gramian $P=\Psi^{*} \Psi$, which satisfies $A^{*} P+P A=-C^{*} C$. For more on Gramians we refer to Hansen and Weiss [11] or Russell and Weiss [23].

DeFINITION 2.1. With the notation as in (2.11) the pair $(A, C)$ is exactly observable in time $T$ if $\Psi_{T}$ is bounded from below, i.e., there exists $k_{T}>0$ such that

$$
\int_{0}^{T}\left\|C \mathbb{T}_{t} z_{0}\right\|_{Y}^{2} d t \geq k_{T}^{2}\left\|z_{0}\right\|_{X}^{2} \quad \forall z_{0} \in \mathcal{D}(A) .
$$

The pair $(A, C)$ is approximately observable in time $T$ if $\operatorname{Ker} \Psi_{T}=\{0\}$.

As is well known, for finite-dimensional systems the properties in Definition 2.1 are equivalent and independent of $T$, and if they hold, then we say that $(A, C)$ is observable. We remark that $\int_{0}^{T}\left\|C \mathbb{T}_{t} z_{0}\right\|_{Y}^{2}>0 \forall z_{0} \in \mathcal{D}(A)$ is not sufficient for approximate observability in time $T$.

Clearly, the following assertions hold true.

Proposition 2.2. The pair $(A, C)$ is exactly observable in time $T$ if and only if $P_{T}$ is invertible. Similarly, $(A, C)$ is approximately observable in time $T$ if and only if $P_{T}$ is one-to-one. If $T>\tau$, then $P_{T} \geq P_{\tau}$.

With the notation from (2.11) it is easy to see that if $z_{0} \in \mathcal{D}(A)$, then $\Psi_{T} z_{0} \in H^{1}(0, T ; Y)$. The following partial converse will be needed in section 3 .

Proposition 2.3. With the notation as in (2.11), suppose that $(A, C)$ is exactly observable in time $T_{0}$. If $z_{0} \in X$ and $T>T_{0}$ are such that $\Psi_{T} z_{0} \in H^{1}(0, T ; Y)$, then $z_{0} \in \mathcal{D}(A)$. For $T=T_{0}$, the implication is not true in general.

Proof. Denote $y=\Psi_{T} z_{0}$, so that $y \in H^{1}(0, T ; Y)$. Using, for example, Proposition VIII.3 (p. 124) in Brezis [6], we obtain

$$
\sup _{\varepsilon \in\left(0, T-T_{0}\right)} \int_{0}^{T_{0}}\left\|\frac{y(t+\varepsilon)-y(t)}{\varepsilon}\right\|_{Y}^{2} d t<\infty .
$$

Since, for almost every $t \in\left[0, T_{0}\right], y(t+\varepsilon)-y(t)=C_{\Lambda} \mathbb{T}_{t}\left(\mathbb{T}_{\varepsilon}-I\right) z_{0}$, it follows that

$$
\sup _{\varepsilon \in\left(0, T-T_{0}\right)}\left\|\Psi_{T_{0}} \frac{\mathbb{T}_{\varepsilon}-I}{\varepsilon} z_{0}\right\|_{L^{2}\left(\left[0, T_{0}\right], Y\right)}<\infty .
$$

Because of the exact observability estimate (2.12), this implies

$$
\sup _{\varepsilon \in\left(0, T-T_{0}\right)}\left\|\frac{\mathbb{T}_{\varepsilon}-I}{\varepsilon} z_{0}\right\|_{X}<\infty .
$$

By a simple result on operator semigroups, see for instance Theorem 2.12 (p. 88) in Butzer and Berens [7], it follows that $z_{0} \in \mathcal{D}(A)$. To see that for $T=T_{0}$ the implication is false, consider the left-shift semigroup $\mathbb{T}$ on $X=L^{2}[0,1]$ with point observation at the left end. Thus $A=\frac{d}{d \xi}, \mathcal{D}(A)=\left\{x \in H^{1}(0,1) \mid x(1)=0\right\}$, and $C x=x(0)$. This system is exactly observable in time $T_{0}=1$. However, if $z_{0}(\xi)=$ $1 \forall \xi \in(0,1)$, then $\Psi_{1} z_{0} \in H^{1}(0,1)$, but $z_{0} \notin \mathcal{D}(A)$.

Definition 2.4. Let $A$ be the generator of a strongly continuous semigroup $\mathbb{T}$ on $X$ and let $B \in \mathcal{L}\left(U, X_{-1}\right)$ be an admissible control operator for $\mathbb{T}$. The pair $(A, B)$ is exactly controllable in time $T>0$, if for every $f_{0} \in X$ there exists a $u \in L^{2}([0, T], U)$ such that

$$
\int_{0}^{T} \mathbb{T}_{T-\sigma} B u(\sigma) d \sigma=f_{0} .
$$


$(A, B)$ is approximately controllable in time $T$ if the set of those $f_{0}$ for which the above property holds is dense.

In other words, we say that $(A, B)$ is exactly controllable in time $T$ if $\Phi_{T}$ is onto, i.e., $\operatorname{Ran} \Phi_{T}=X$, and $(A, B)$ is approximately controllable in time $T$ if $\operatorname{Ran} \Phi_{T}$ is dense in $X$. For finite-dimensional systems the above properties are equivalent and independent of $T$, and if they hold we say that $(A, B)$ is controllable.

Proposition 2.5. We assume that $A$ is the generator of a semigroup $\mathbb{T}$ on $X$ and $B \in \mathcal{L}\left(U, X_{-1}\right)$ is an admissible control operator for $\mathbb{T}$. Then $(A, B)$ is exactly controllable in time $T$ if and only if $\left(A^{*}, B^{*}\right)$ is exactly observable in time T. Similarly, $(A, B)$ is approximately controllable in time $T$ if and only if $\left(A^{*}, B^{*}\right)$ is approximately observable in time $T$.

This proposition is an easy consequence of (2.10). It is used frequently in the literature on control of systems governed by PDEs (see, e.g., the HUM method of Lions [20]). For more details on exact controllability (observability) in a functionalanalytic setting we refer to Avdonin and Ivanov [2] or [23] and the references therein. In the PDE's-setting, the relevant literature is overwhelming, and we mention the books of Lions [20], Lagnese and Lions [16], and Komornik [21] and the paper of Bardos, Lebeau, and Rauch [5].

3. Main results. First we give the definition of the simultaneous controllability concepts used.

DeFinition 3.1. For $j \in\{1,2\}$, let $A_{j}$ be the generators of the strongly continuous semigroups $\mathbb{T}^{j}$ acting on the Hilbert spaces $X^{j}$. Let $U$ be a Hilbert space and let $B_{j} \in \mathcal{L}\left(U, X_{-1}^{j}\right)$ be admissible control operators for $\mathbb{T}^{j}$.

The pairs $\left(A_{j}, B_{j}\right)$ are called simultaneously exactly controllable in time $T>0$ if for every state $f_{j} \in X^{j}$ there exists a function $u \in L^{2}([0, T], U)$ such that

$$
\int_{0}^{T} \mathbb{T}_{T-\sigma}^{j} B_{j} u(\sigma) d \sigma=f_{j}
$$

The same pairs are called simultaneously approximately controllable in time $T>0$ if the property described above holds for $\left(f_{1}, f_{2}\right)$ in a dense subspace of $X^{1} \times X^{2}$.

It is clear that the concepts introduced in the last definition are equivalent to the exact (approximate) controllability in time $T$ of the pair

$$
A=\left[\begin{array}{cc}
A_{1} & 0 \\
0 & A_{2}
\end{array}\right], \quad B=\left[\begin{array}{l}
B_{1} \\
B_{2}
\end{array}\right] .
$$

Using Proposition 2.5, the above concepts can be characterized by duality.

Proposition 3.2. With the notation of Definition 3.1, we have:

1. The pairs $\left(A_{1}, B_{1}\right)$ and $\left(A_{2}, B_{2}\right)$ are simulteanously exactly controllable in time $T$ if and only if there exists $k_{T}>0$ such that $\forall\left(z_{0}^{1}, z_{0}^{2}\right) \in \mathcal{D}\left(A_{1}^{*}\right) \times \mathcal{D}\left(A_{2}^{*}\right)$ we have

$$
\int_{0}^{T}\left\|B_{1}^{*} \mathbb{T}_{t}^{1 *} z_{0}^{1}+B_{2}^{*} \mathbb{T}_{t}^{2 *} z_{0}^{2}\right\|_{U}^{2} \geq k_{T}^{2}\left(\left\|z_{0}^{1}\right\|_{X^{1}}^{2}+\left\|z_{0}^{2}\right\|_{X^{2}}^{2}\right) .
$$

2. The pairs $\left(A_{1}, B_{1}\right)$ and $\left(A_{2}, B_{2}\right)$ are simultaneously approximately controllable in time $T$ if and only if the following statement holds.

$$
\text { If }\left(z_{0}^{1}, z_{0}^{2}\right) \in X^{1} \times X^{2} \text { are such that }
$$

$$
B_{1 \Lambda}^{*} \mathbb{T}_{t}^{1 *} z_{0}^{1}+B_{2 \Lambda}^{*} \mathbb{T}_{t}^{2 *} z_{0}^{2}=0 \quad \text { for almost every } t \in[0, T],
$$




$$
\text { then }\left(z_{0}^{1}, z_{0}^{2}\right)=(0,0) \text {. }
$$

We mention that in (3.2) we must use the $\Lambda$-extensions as in (2.10). The reason is that it is not possible to use only $\left(z_{0}^{1}, z_{0}^{2}\right) \in \mathcal{D}\left(A_{1}^{*}\right) \times \mathcal{D}\left(A_{2}^{*}\right)$ (this follows from the comments after Definition 2.1).

The main result of this section is the following theorem.

THEOREM 3.3. Let $A$ be the generator of the strongly continuous semigroup $\mathbb{T}$ acting on the Hilbert space $X$. Let $B \in \mathcal{L}\left(\mathbb{C}^{m}, X\right)$ be an admissible control operator for $\mathbb{T}$ and assume that $(A, B)$ is exactly controllable in time $T_{0}$. Let $a \in \mathbb{C}^{n \times n}$ and $b \in \mathbb{C}^{n \times m}$ be matrices such that $(a, b)$ is controllable. Assume that $A$ and a have no common eigenvalues. Then the pairs $(A, B)$ and $(a, b)$ are simultaneously exactly controllable in any time $T>T_{0}$.

First we prove the following approximate controllability result.

Lemma 3.4. Suppose that $T>T_{0}$ and that $(A, B),(a, b)$ satisfy the assumptions of Theorem 3.3. Then these two pairs are simultaneously approximately controllable in time $T$ for every $T>T_{0}$.

Proof. Let $T>T_{0}$ be fixed. Denote by $V$ the set of all $v_{0} \in \mathbb{C}^{n}$ such that there exists a $z_{0} \in X$ with

$$
B_{\Lambda}^{*} \mathbb{T}_{t}^{*} z_{0}+b^{*} e^{a^{*} t} v_{0}=0 \quad \text { for almost every } t \in[0, T] .
$$

Using the approximate controllability of $(A, B)$ in time $T_{0}$ and Proposition 2.5, we see that the function $t \rightarrow B_{\Lambda}^{*} \mathbb{T}_{t}^{*} z_{0}, t \in[0, T]$, determines $z_{0}$. By (3.3), this function is determined by $v_{0}$. Thus, if $v_{0} \in V$, then $z_{0}$ satisfying (3.3) is unique and depends linearly on $v_{0}: z_{0}=Q v_{0}$. Since the function $t \rightarrow b^{*} e^{a^{*} t} v_{0}$ is smooth, by Proposition 2.3 we have that

$$
Q v_{0} \in \mathcal{D}\left(A^{*}\right) \quad \forall v_{0} \in V .
$$

Now we show that $\forall v_{0} \in V$, we have

$$
Q a^{*} v_{0}=A^{*} Q v_{0} .
$$

Indeed, by differentiating (3.3) with respect to time and using (2.8), we obtain that

$$
B_{\Lambda}^{*} \mathbb{T}_{t}^{*} A^{*} Q v_{0}+b^{*} e^{a^{*} t} a^{*} v_{0}=0
$$

for almost every $t \in[0, T]$, which shows that $a^{*} v_{0} \in V$ and (3.4) holds.

Let $\widetilde{a}$ denote the restriction of $a^{*}$ to its invariant subspace $V$. If $V \neq\{0\}$, then $\widetilde{a}$ must have an eigenvalue $\lambda \in \sigma\left(a^{*}\right)$ and a corresponding eigenvector $\widetilde{v}$. Formula (3.4) implies that $A^{*} Q \widetilde{v}=\lambda Q \widetilde{v}$. Since $Q$ is one-to-one, we have that $Q \widetilde{v} \neq 0$, so that $\lambda$ is an eigenvalue of $A^{*}$. This is in contradiction to the assumption in Theorem 3.3, and hence we must have $V=\{0\}$. Thus, (3.3) implies that $\left(z_{0}, v_{0}\right)=(0,0)$ and we can apply the second part of Proposition 3.2.

Proof of Theorem 3.3. Let $T>T_{0}$ be fixed. According to Proposition 2.5 it suffices to show that the pair

$$
\mathcal{A}^{*}=\left[\begin{array}{cc}
A^{*} & 0 \\
0 & a^{*}
\end{array}\right], \quad \mathcal{B}^{*}=\left[\begin{array}{ll}
B^{*} & b^{*}
\end{array}\right]
$$

is exactly observable in time $T$. We already know from Lemma 3.4 and Proposition 2.5 that $\left(\mathcal{A}^{*}, \mathcal{B}^{*}\right)$ is approximately observable in time $T$. Let $\mathcal{P}_{T}$ denote the observability 
Gramian of $\left(\mathcal{A}^{*}, \mathcal{B}^{*}\right)$, so that $\mathcal{P}_{T}>0$. We partition $\mathcal{P}_{T}$ in a natural way, according to the product space $X \times \mathbb{C}^{n}$ :

$$
\mathcal{P}_{T}=\left[\begin{array}{cc}
P_{T} & L \\
L^{*} & p_{T}
\end{array}\right]
$$

We want to show that $\mathcal{P}_{T}$ is invertible (i.e., bounded from below). It is not difficult to see that $P_{T}$ is the observability Gramian of $\left(A^{*}, B^{*}\right)$ and $p_{T}$ is the observability Gramian of $\left(a^{*}, b^{*}\right)$. As $\left(A^{*}, B^{*}\right)$ and $\left(a^{*}, b^{*}\right)$ are exactly observable in time $T$, by Proposition 2.2, both $P_{T}$ and $p_{T}$ are positive and boundedly invertible. We bring in the Schur-type factorization

$$
\left[\begin{array}{cc}
P_{T} & L \\
L^{*} & p_{T}
\end{array}\right]=\left[\begin{array}{cc}
P_{T} & 0 \\
L^{*} & I
\end{array}\right]\left[\begin{array}{cc}
P_{T}^{-1} & 0 \\
0 & \Delta
\end{array}\right]\left[\begin{array}{cc}
P_{T} & L \\
0 & I
\end{array}\right],
$$

where $\Delta=p_{T}-L^{*} P_{T}^{-1} L$ (this is checked by multiplying out). Notice that the first factor is the adjoint of the last, and they are invertible. Therefore, $\mathcal{P}_{T}$ is invertible if and only if the middle factor is invertible. Since $P_{T}^{-1}$ is obviously bounded from below, we see that $\mathcal{P}_{T}$ is bounded from below if and only if $\Delta$ is bounded from below. Since $\mathcal{P}_{T}>0$, from the factorization we see that $\Delta>0$. But $\Delta$ is a matrix, so that $\Delta>0$ implies that $\Delta$ is invertible. Thus we have proved that $\mathcal{P}_{T}$ is invertible. By Proposition $2.2,\left(\mathcal{A}^{*}, \mathcal{B}^{*}\right)$ is exactly observable in time $T$.

Remark 3.5. Under the assumptions of Theorem 3.3, in general, the two systems will not be simultaneously exactly controllable in time $T_{0}$. An example to illustrate this will be given in section 4 .

In the rest of this section we shall investigate simultaneous approximate controllability. With the assumptions of Theorem 3.3 we obviously obtain simultaneous approximate controllability, but the result is not sharp as it asks for exact controllability of each component. We give below a simultaneous approximate controllability result by supposing only approximate controllability of each component.

At this point we introduce some notation. Let $A$ be the generator of a strongly continuous semigroup. Then the resolvent set $\rho(A)$ contains a right half-plane. The resolvent set is not necessarily connected, and we denote by $\rho_{\infty}(A)$ the connected component of $\rho(A)$ which contains some right half-plane. (Obviously, there is only one such component.) In particular, if $\sigma(A)$ is countable, as is often the case in applications, then $\rho_{\infty}(A)=\rho(A)$.

Proposition 3.6. Let $A$ be the generator of the strongly continuous semigroup $\mathbb{T}$ acting on the Hilbert space $X$. Let $B \in \mathcal{L}\left(\mathbb{C}^{m}, X_{-1}\right)$ be an admissible control operator for $\mathbb{T}$ and assume that $(A, B)$ is approximately controllable in time $T_{0}$. Let $a \in \mathbb{C}^{n \times n}$ and $b \in \mathbb{C}^{n \times m}$ be matrices such that $(a, b)$ is controllable. Further, assume that

$$
\sigma(a) \subset \rho_{\infty}(A) .
$$

Then there exists $T>0$ such that the pairs $(A, B)$ and $(a, b)$ are simultaneously approximately controllable in time $T$.

Proof. To arrive at a contradiction, we assume that the opposite holds: $(\mathcal{A}, \mathcal{B})$ from (3.6) is not approximately controllable in any time. Then it follows from Proposition 3.2 that for every $k \in \mathbb{N}$ there exists a $z_{k} \in X$ and a $v_{k} \in \mathbb{C}^{n}$ such that $\left(z_{k}, v_{k}\right) \neq(0,0)$ and

$$
B_{\Lambda}^{*} \mathbb{T}_{t}^{*} z_{k}+b^{*} e^{a^{*} t} v_{k}=0 \quad \forall t \in[0, k]
$$


It follows from the approximate observability in time $T_{0}$ of $\left(A^{*}, B^{*}\right)$ that $\forall k>T_{0}$ we must have $v_{k} \neq 0$. Hence we may assume without loss of generality that $\left\|v_{k}\right\|_{\mathbb{C}^{n}}=1$. By the compactness of the unit ball in $\mathbb{C}^{n}$, we may assume further that the sequence $\left(v_{k}\right)$ is convergent: $\lim v_{k}=v_{0}$. Then it follows that if we define the functions $y_{k} \in L_{l o c}^{2}\left([0, \infty), \mathbb{C}^{m}\right)$ by

$$
y_{k}(t)=b^{*} e^{a^{*} t} v_{k} \quad \text { for } k \in\{0,1,2, \ldots\},
$$

then $\lim y_{k}=y_{0}\left(\right.$ in $\left.L_{l o c}^{2}\right)$. Let $\Psi_{T_{0}}$ be the operator defined by

$$
\Psi_{T_{0}} z_{0}=B_{\Lambda}^{*} \mathbb{T}_{t}^{*} z_{0} \quad \forall t \in\left[0, T_{0}\right]
$$

and let $\Pi_{T_{0}}$ denote the truncation of a function defined on $[0, \infty)$ to $\left[0, T_{0}\right]$. Then (3.8) implies that

$$
\Psi_{T_{0}} z_{k}+\Pi_{T_{0}} y_{k}=0 \quad \forall k \geq T_{0} .
$$

Since Ker $\Psi_{T_{0}}=\{0\}$, the above equation shows that $z_{k}$ is uniquely determined by $y_{k}$, which in turn is obtained from $v_{k}$. All these dependencies are linear, so that there is an operator $R: \mathbb{C}^{n} \rightarrow X$ (possibly nonunique, depending on the span of all $\left.v_{k}\right)$ such that $z_{k}=R v_{k} \forall k \in \mathbb{N}$. Hence, the sequence $\left(z_{k}\right)$ is convergent, and we put $z_{0}=\lim z_{k}=R v_{0}$. Now it is easy to conclude from (3.8) that

$$
\left(\Psi z_{0}\right)(t)+b^{*} e^{a^{*} t} v_{0}=0 \quad \text { for almost every } t \geq 0 .
$$

Taking Laplace transforms, we obtain from the last formula that for some $\alpha \in \mathbb{R}$ and every $s \in \mathbb{C}$ with $\operatorname{Re} s>\alpha$,

$$
B^{*}\left(s I-A^{*}\right)^{-1} z_{0}+b^{*}\left(s I-a^{*}\right)^{-1} v_{0}=0 .
$$

By analytic continuation, this formula remains valid on $\rho_{\infty}\left(A^{*}\right) \backslash \sigma\left(a^{*}\right)$. (On the other connected components of $\rho\left(A^{*}\right)$ we have no such information.) Since $v_{0} \neq 0$ (actually, its norm is 1) and $\left(a^{*}, b^{*}\right)$ is observable, the rational function $b^{*}\left(s I-a^{*}\right)^{-1} v_{0}$ is not zero. Therefore it has poles at a nonempty subset of $\sigma\left(a^{*}\right)$, which by $(3.7)$ is contained in $\rho_{\infty}\left(A^{*}\right)$. The first term in (3.9) being analytic around $\sigma\left(a^{*}\right)$, it follows that the left-hand side of (3.9) has poles, which is absurd. Thus we have proved that $(\mathcal{A}, \mathcal{B})$ must be approximately controllable in some time $T$.

Note that the lemma says nothing about the time $T$ in which $(\mathcal{A}, \mathcal{B})$ is approximately controllable. If $T_{0}$ is minimal for $(A, B)$, then of course $T \geq T_{0}$.

In the last part of this section we characterize the reachable subspaces of an exactly controllable system, when the input function is restricted to Sobolev type spaces strictly included in $L^{2}$.

Let $A$ be the generator of a strongly continuous semigroup $\mathbb{T}$ on $X$ and let $B \in$ $\mathcal{L}\left(U, X_{-1}\right)$ be an admissible control operator for $\mathbb{T}$. Suppose that the pair $(A, B)$ is exactly controllable in time $T$, in the sense of Definition 2.4. This means that the range of the operator $\Phi_{T}$ defined by (2.4) is equal to $X$. A natural question is the characterization of the states which can be reached by more regular inputs. Define

$$
H_{L}^{1}(0, T ; U)=\left\{\psi \in H^{1}(0, T ; U) \mid \psi(0)=0\right\} .
$$

The existence and uniqueness result below shows that the space reachable by means of controls in $H_{L}^{1}(0, T ; U)$ cannot be larger than the space $Z$ defined by

$$
Z=X_{1}+(\beta I-A)^{-1} B U=(\beta I-A)^{-1}(X+B U),
$$


where $\beta \in \rho(A)$ ( $Z$ does not depend on the choice of $\beta$ ). The norm on $Z$ is defined by

$$
\|z\|_{Z}^{2}=\inf \left\{\|x\|^{2}+\|u\|^{2} \mid x \in X, u \in U, z=(\beta I-A)^{-1}(x+B u)\right\} .
$$

Lemma 3.7. For any $u \in H_{L}^{1}(0, T ; U)$, the solution $z$ of $(2.2)$ with $z(0)=0$ is such that

$$
z \in C(0, T ; Z) \cap C^{1}(0, T, X) .
$$

Proof. Let $u \in H_{L}^{1}(0, T ; U)$ and denote by $w$ the solution of

$$
\dot{w}=A w+B \dot{u}, \quad w(0)=0 .
$$

As $B$ is an admissible control operator we have that $w \in C([0, T] ; X)$. Moreover it is easily checked that the function $t \rightarrow \int_{0}^{T} w(s) d s$ satisfies (2.2). Since the solution of (2.2) with $z(0)=0$ is unique, we obtain

$$
z(t)=\int_{0}^{T} w(s) d s
$$

which obviously yields that

$$
z \in C^{1}([0, T], X)
$$

On the other hand (2.2) gives

$$
(\beta I-A) z(t)=\beta z(t)-\dot{z}(t)+B u(t) \quad \forall t \in[0, T] .
$$

Since $\beta z-\dot{z}+B u \in C([0, T], X+B U)$, relation (3.12) with $\beta \in \rho(A)$ implies

$$
z \in C([0, T], Z) \text {. }
$$

From (3.11) and (3.13) we clearly obtain the conclusion of the lemma.

We can now characterize the states which are reachable by means of input functions in $H_{L}^{1}(0, T ; U)$ as follows.

Proposition 3.8. Suppose that the pair $(A, B)$ is exactly controllable in time $T_{0}$. Then $\forall T>T_{0}$, the reachable space by means of input functions $u \in H_{L}^{1}(0, T ; U)$ is the space $Z$ from (3.10).

Proof. We know from Lemma 3.7 that the reachable space is included in $Z$. To show that $Z$ is contained in the reachable space, take $\beta \in \rho(A)$ and consider two systems with states $w$ and $v$ and input $u_{1}$, described by

$$
\begin{gathered}
\dot{w}=(A-\beta I) w+B u_{1}, \\
\dot{v}=u_{1} .
\end{gathered}
$$

For an arbitrary $z^{0} \in Z$ choose $w^{0} \in X, v^{0} \in U$ such that

$$
z^{0}=(\beta I-A)^{-1}\left[w^{0}-B v^{0}\right] .
$$

Since 0 is not an eigenvalue of $A-\beta I$, by Theorem 3.3 the systems (3.14) and (3.15) are simultaneously exactly controllable in any time $T>T_{0}$. Hence we can find $u_{1} \in L^{2}([0, T] ; U)$ such that the solutions $w, v$ of (3.14) and (3.15) satisfy

$$
w(0)=0, \quad w(T)=e^{-\beta T} w^{0}, \quad v(0)=0, \quad v(T)=e^{-\beta T} v^{0} .
$$


We define the function $z_{1}$ by

$$
z_{1}(t)=(\beta I-A)^{-1}(w(t)-B v(t)) \quad \forall t \in[0, T] .
$$

Then it is easy to see that

$$
z_{1}(0)=0, \quad z_{1}(T)=e^{-\beta T} z^{0} .
$$

Moreover, after a simple calculation, (3.14) and (3.15) imply that

$$
\dot{z}_{1}(T)=-w(t)=(A-\beta I) z_{1}(t)-B v(t) \quad \forall t \in(0, T) .
$$

If we define now

$$
z(t)=e^{\beta t} z_{1}(t), \quad u(t)=e^{\beta t} v(t),
$$

relations (3.18) and (3.19) imply that $z$ and $u$ satisfy (2.2) together with $z(0)=0$ and $z(T)=z^{0}$. This means that $Z$ is included in the space reachable by means of input functions $u \in H_{L}^{1}(0, T ; U)$, as claimed.

\section{Applications.}

4.1. Applications to the equation of a vibrating string. In this subsection we apply the results obtained in previous sections to the equation of a nonhomogeneous vibrating string. First we show that, with suitably chosen spaces, the system corresponding to the string equation and an integrator are simultaneously exactly controllable. In the case of a homogeneous string we show that the simultaneous exact controllability time is strictly larger than the exact controllability time for the string alone, i.e., we give the counterexample announced in Remark 3.5. In the second part of this subsection we characterize the space of the states which are reachable by means of an $H^{1}$ or $H^{2}$ input function $u$ with $u(0)=0$ and, in the case $u \in H^{2}$, also $\dot{u}(0)=0$.

Let us consider the initial and boundary value problem

$$
\begin{cases}\ddot{w}(x, t)=\left[m(x) w_{x}(x, t)\right]_{x}, & 0<x<1 \\ w(0, t)=0, & w(1, t)=u(t), \\ w(x, 0)=0, & \dot{w}(x, 0)=0\end{cases}
$$

with

$$
m \in W^{1, \infty}(0,1), \quad m(x) \geq m_{0}>0 \quad \forall x \in(0,1) .
$$

The equations above represent the simplest model of a nonhomogeneous elastic string. Following well-known ideas (see for instance Lasiecka and Triggiani [18], [19]) the system (4.1) can be written in the abstract form (2.2), provided we use the notation

$$
\begin{gathered}
z=\left[\begin{array}{c}
w \\
\dot{w}
\end{array}\right], \quad X=L^{2}[0,1] \times H^{-1}(0,1), \quad U=\mathbb{C}, \\
A=\left[\begin{array}{cc}
0 & I \\
A_{0} & 0
\end{array}\right], \quad B=\left[\begin{array}{c}
0 \\
-A_{0} D
\end{array}\right],
\end{gathered}
$$


where

$$
\mathcal{D}\left(A_{0}\right)=H_{0}^{1}(0,1), \quad A_{0}: \mathcal{D}\left(A_{0}\right) \rightarrow H^{-1}(0,1), \quad A_{0} h=\left(m(x) h_{x}\right)_{x},
$$

so that $A_{0}<0$, and the Dirichlet map $D: \mathbb{C} \rightarrow L^{2}[0,1]$ is defined by

$$
D \alpha=y \Longleftrightarrow\left\{\left(m(x) y_{x}\right)_{x}=0 \text { in }(0,1), y(0)=0, y(1)=\alpha\right\}
$$

(see also [1]). From the above it clearly follows that $A: \mathcal{D}(A) \rightarrow X$, with

$$
\mathcal{D}(A)=H_{0}^{1}(0,1) \times L^{2}[0,1],
$$

and that $A$ is skew-adjoint: $A^{*}=-A$. Note that $B^{*}=\left[\begin{array}{ll}0 & D^{*}\end{array}\right]$ and, for every $h \in H^{2}(0,1) \cap H_{0}^{1}(0,1), \quad D^{*} A_{0} h=m(1) h_{x}(1)$. We denote by $\mathbb{T}$ the semigroup generated by $A$. Well-known computations, using the above expressions for $A^{*}$ and $B^{*}$ (see again [18], [19]) give that

$$
B^{*} \mathbb{T}_{t}^{*}\left[\begin{array}{l}
z^{0} \\
z^{1}
\end{array}\right]=m(1) \phi_{x}(1, t) \quad \forall\left[\begin{array}{l}
z^{0} \\
z^{1}
\end{array}\right] \in \mathcal{D}(A),
$$

where $\phi$ solves the corresponding homogeneous problem

$$
\begin{gathered}
\ddot{\phi}(x, t)=\left(m(x) \phi_{x}(x, t)\right)_{x}, \quad 0<x<1, t \in(0, T), \\
\phi(0, t)=\phi(1, t)=0, \quad t \in[0, T], \\
\phi(\cdot, 0)=\phi^{0}=A_{0}^{-1} z^{1} \in H^{2}(0,1) \cap H_{0}^{1}(0,1), \\
\dot{\phi}(\cdot, 0)=\phi^{1}=z^{0} \in H_{0}^{1}(0,1) .
\end{gathered}
$$

It is by now well known that $B$ is an admissible control operator and the couple $(A, B)$ is exactly controllable in any time $T>T_{0}$, where $T_{0}=\frac{2}{\sqrt{m_{0}}}$ (see for instance Zuazua [27]). Moreover, if $m=1$, then the system $(A, B)$ is exactly controllable in time 2 (see for instance Haraux [15]).

Consider now the following system of two scalar differential equations with the same input $u$ :

$$
\left\{\begin{array}{l}
\dot{v}=u \\
\dot{w}=w+u .
\end{array}\right.
$$

The result below, concerning the simultaneous exact controllability of (4.1) and (4.9), gives, in particular, the counterexample announced in Remark 3.5.

Proposition 4.1. The systems (4.1) and (4.9) are simultaneously exactly controllable in any time $T>T_{0}$, where $T_{0}=\frac{2}{\sqrt{m_{0}}}$. However, if $m=1$, then the systems (4.1) and (4.9) are not simultaneously approximately controllable in time $T_{0}=2$.

Proof. We can write the system (4.9) in the form $\dot{q}=a q+b u$, where

$$
q=\left[\begin{array}{c}
v \\
w
\end{array}\right], \quad a=\left[\begin{array}{ll}
0 & 0 \\
0 & 1
\end{array}\right], \quad b=\left[\begin{array}{l}
1 \\
1
\end{array}\right]
$$


and it is clear that $(a, b)$ is controllable. The eigenvalues of $A$ from (4.3) are on the imaginary axis and nonzero. The simultaneous exact controllability in any time $T>T_{0}$ follows from the exact controllability of the system in (4.1) in any time $T>T_{0}$, by applying Theorem 3.3 .

We still have to prove the lack of simultaneous approximate controllability in time 2 , in the case of a homogeneous string with $m=1$. Choose $w_{0} \in \mathbb{R}, w_{0} \neq 0$. As the family formed by $\left(\sin (n \pi t)_{n \geq 1}, \cos (n \pi t)_{n \geq 1}\right)$ together with the constant function $1 / \sqrt{2}$ is an orthonormal basis in $L^{2}(0,2)$, we can find sequences $\left(a_{n}\right)_{n \geq 1}$ and $\left(b_{n}\right)_{n \geq 1}$ in $l^{2}$ and $v_{0} \in \mathbb{R}$ such that

$$
\sum_{n=1}^{\infty}(-1)^{n}\left[a_{n} \cos (n \pi t)+b_{n} \sin (n \pi t)\right]+v_{0}+e^{t} w_{0}=0 \quad \text { for a.e. } t \in[0,2] .
$$

Note that the functions $\sin (n \pi x)(x \in(0,1))$ are eigenvectors of $A_{0}$. If we denote

$$
z^{0}(x)=\pi \sum_{n=1}^{\infty} b_{n} \sin (n \pi x), \quad z^{1}(x)=\pi^{2} \sum_{n=1}^{\infty} n a_{n} \sin (n \pi x),
$$

then $z^{0} \in L^{2}[0,1]$ and $z^{1} \in H^{-1}(0,1)$. Now using (4.4) and (4.10), relation (4.11) can be written as

$$
B_{\Lambda}^{*} \mathbb{T}_{t}^{*}\left[\begin{array}{c}
z^{0} \\
z^{1}
\end{array}\right]+b^{*} e^{a^{*} t}\left[\begin{array}{c}
v_{0} \\
w_{0}
\end{array}\right]=0 \quad \text { for almost every } t \in[0,2] .
$$

Since $w_{0} \neq 0$, this relation with Proposition 3.2 implies that the systems (4.1) and (4.9) are not simultaneously approximately controllable in time $T_{0}=2$.

For $l>0$ we define the space

$$
H_{L}^{2}(0, l)=\left\{u \in H^{2}(0, l) \mid u(0)=\dot{u}(0)=0\right\} .
$$

The states of the system (4.1) which can be reached by means of $H_{L}^{1}$ and $H_{L}^{2}$ input functions can be characterized as follows.

Proposition 4.2. Suppose that $m(x)$ satisfies (4.2) and $T>T_{0}=\frac{2}{\sqrt{m_{0}}}$. Then the space of all states $(w(T), \dot{w}(T))$ which can be reached in time $T$ by means of input functions $u \in H_{L}^{1}(0, T)$ is $Z=H_{L}^{1}(0,1) \times L^{2}[0,1]$.

Moreover, the space of all states $(w(T), \dot{w}(T))$ which can be reached in time $T$ by means of input functions $u \in H_{L}^{2}(0, T)$ is $Z_{1}=\left[H_{L}^{1}(0,1) \cap H^{2}(0,1)\right] \times H_{L}^{1}(0,1)$.

Proof. For $u \in H_{L}^{1}(0, T)$ it suffices to apply Proposition 3.8 and to notice that, with the notation (4.3), the space $Z$ defined by $(3.10)$ is $H_{L}^{1}(0,1) \times L^{2}[0,1]$. For $u \in H_{L}^{2}(0, T)$ we consider the new input $\widetilde{u}=\dot{u}$, a new state space equal to $H_{L}^{1}(0,1) \times$ $L^{2}[0,1]$, and we apply again Proposition 3.8 .

4.2. Controllability of a coupled system. Consider a vertical string whose horizontal displacement in a given plane is described by the wave equation on the spatial domain $(0,1)$. The upper end (corresponding to $x=0$ ) is kept fixed and an object of mass $M$ is attached at the lower end (corresponding to $x=1$ ). The external input is a horizontal force $v$ acting on the object, and it is contained in the plane mentioned earlier. We neglect the moment of inertia of the object (i.e., we imagine the object to be very small). From simple physical considerations, and taking a certain constant to be one, we obtain that this system is described by the following equations, 
valid $\forall x \in(0,1)$ and $\forall t \in(0, \infty)$ :

$$
\left\{\begin{array}{l}
\ddot{w}(x, t)=\left[m(x) w_{x}\right]_{x}(x, t), w(0, t)=0, \\
M \ddot{w}(1, t)-w_{x}(1, t)=v(t), \\
w(x, 0)=\dot{w}(x, 0)=0, x \in(0,1) .
\end{array}\right.
$$

Here, $w$ is the controlled wave (horizontal displacement) and $\dot{w}$ is the horizontal velocity. The appropriate spaces for all these functions will be specified later. The point $x=0$ is just reflecting waves, while the active end $x=1$ is where both the observation and the control take place. We shall often write $w(t)$ to denote a function of $x$, meaning that $w(t)(x)=w(x, t)$, and similarly for other functions.

A direct analysis of the well-posedness, controllability, and observability of this system is not trivial, in spite of the simplicity of the system. We will show below that we can obtain a sharp result by simply applying Proposition 4.2 . We begin by identifying the natural state space of (4.12).

Proposition 4.3. Suppose that $m(\cdot)$ satisfies (4.2) and that $v \in L^{2}[0, T]$. Then the initial and boundary value problem (4.12) admits a unique solution

$$
w \in C\left(0, T ; H_{L}^{1}(0,1) \cap H^{2}(0,1)\right) \cap C^{1}\left(0, T ; H_{L}^{1}(0,1)\right) .
$$

Proof. Using semigroups or a standard Galerkin method, it is easy to prove that $\forall v \in L^{2}[0, T]$, the problem (4.12) admits a unique solution

$$
w \in C\left(0, T ; H_{L}^{1}(0,1)\right) \cap C^{1}\left(0, T ; L^{2}[0,1]\right),
$$

which satisfies the first equation from $(4.12)$ in $\mathcal{D}^{\prime}((0,1) \times(0, T))$ and the second in $\mathcal{D}^{\prime}(0, T)$ (notice that $w_{x}(1, \cdot)$ makes sense in $\left.H^{-2}(0, T)\right)$. Consider a sequence $\left(v_{n}\right)$ in $\mathcal{D}(0, T)$ such that $v_{n} \rightarrow v$ in $L^{2}[0, T]$. If we denote by $\left(w_{n}\right)$ the corresponding sequence of smooth solutions of (4.12), it is clear that

$$
\begin{gathered}
w_{n} \rightarrow w \quad \text { in } L^{\infty}\left(0, T ; H_{L}^{1}(0,1)\right) \cap W^{1, \infty}\left(0, T ; L^{2}[0,1]\right), \\
w_{n}(1, t)=\dot{w}_{n}(1, t)=0 \quad \forall n \geq 1 .
\end{gathered}
$$

Moreover, by multiplying the equation

$$
\left(\ddot{w}_{m}-\ddot{w}_{n}\right)(x, t)=\left[m(x)\left(w_{m}-w_{n}\right)_{x}\right]_{x}(x, t)
$$

by $x \frac{\partial}{\partial x}\left(w_{m}-w_{n}\right)(x, t)$ and by integrating over $[0,1] \times[0, T]$, we obtain, after wellknown calculations, the existence of a constant $C>0$ such that

$$
\begin{gathered}
\int_{0}^{T}\left|\left(w_{m}-w_{n}\right)_{x}(1, t)\right|^{2} d t \\
\leq C\left(\left\|w_{n}-w_{m}\right\|_{L^{\infty}\left(0, T ; H^{1}(0,1)\right)}+\left\|\dot{w}_{n}-\dot{w}_{m}\right\|_{L^{\infty}\left(0, T ; L^{2}[0,1]\right)}\right) .
\end{gathered}
$$

Since

$$
M \ddot{w}_{n}(1, t)-\left(w_{n}\right)_{x}(1, t)=v_{n}(t),
$$


relation (4.17) implies that $\ddot{w}_{n}(1, \cdot)$ is a Cauchy sequence in $L^{2}[0, T]$. By using (4.15) and (4.16), we obtain that $w(1, \cdot) \in H_{L}^{2}(0, T)$. The regularity (4.13) follows now from Proposition 4.2.

Proposition 4.4. Suppose that $m$ satisfies (4.2) and $T>T_{0}=\frac{2}{\sqrt{m_{0}}}$. Then the system (4.12) is well posed and exactly controllable in time $T$ in the space $X=$ $\left[H_{L}^{1}(0,1) \cap H^{2}(0,1)\right] \times H_{L}^{1}(0,1)$. In other words, $\left(w^{0}, w^{1}\right) \in\left[H_{L}^{1}(0,1) \cap H^{2}(0,1)\right] \times$ $H_{L}^{1}(0,1)$ if and only if there exists $v \in L^{2}[0, T]$ such that the solution of (4.12) satisfies

$$
w(T)=w^{0}, \quad \dot{w}(T)=w^{1} .
$$

Proof. By Proposition 4.2, for any $\left(w^{0}, w^{1}\right) \in\left[H_{L}^{1}(0,1) \cap H^{2}(0,1)\right] \times H_{L}^{1}(0,1)$ there exist

$$
w \in C\left(0, T ; H^{2}(0,1)\right), \quad u \in H_{L}^{2}(0, T)
$$

satisfying (4.1) and (4.18). From (4.19) it obviously follows that if we define

$$
v(t)=m \ddot{u}(t)-w_{x}(1, t),
$$

then $v \in L^{2}[0, T]$ and $w, v$ satisfy (4.12) and (4.18).

5. The simultaneously reachable subspace of two infinite-dimensional systems. In this section we study an example showing that for certain pairs of infinite-dimensional systems it is still possible to derive results similar to those obtained in the previous section. However, the reachable space and the reachability time are more difficult to characterize. The problem we tackle is the one-dimensional version of an open question raised in Lions [20]. We give here only the results which are simple consequences of recent work on nonharmonic Fourier series. A detailed study of this problem requires new techniques and is the subject of the forthcoming paper by Avdonin and Tucsnak [3].

For $\xi \in(0,1)$ we consider the problems

$$
\left\{\begin{array}{l}
\ddot{w}_{1}(x, t)-\left(w_{1}(x, t)\right)_{x x}=0 \quad \forall x \in(0, \xi), \quad \forall t \in(0, \infty), \\
w_{1}(0, t)=0, w_{1}(\xi, t)=u(t) \quad \forall t \in(0, \infty) \\
w_{1}(x, 0)=0, \dot{w}_{1}(x, 0)=0 \quad \forall x \in(0, \xi)
\end{array}\right.
$$

and

$$
\left\{\begin{array}{l}
\ddot{w}_{2}(x, t)-\left(w_{2}(x, t)\right)_{x x}=0 \quad \forall x \in(\xi, 1), \quad \forall t \in(0, \infty) \\
w_{2}(1, t)=0, w_{2}(\xi, t)=u(t) \quad \forall t \in(0, \infty) \\
w_{2}(x, 0)=0, \dot{w}_{2}(x, 0)=0 \quad \forall x \in(\xi, 1)
\end{array}\right.
$$

The systems above model the vibrations of two strings joined at a common end at $x=\xi$, the input being the displacement of this common point.

By using notation similar to the one used in (4.3), we can easily define the operators $\left(A_{i}, B_{i}\right), i=1,2$ such that the equations (5.1), (5.2) can be written as in (1.1), with state spaces $X^{1}=L^{2}[0, \xi] \times H^{-1}(0, \xi)$ and $X^{2}=L^{2}[\xi, 1] \times H^{-1}(\xi, 1)$. According to classical results, $B_{1}$ (resp., $B_{2}$ ) is an admissible control operator and the system $\left(A_{1}, B_{1}\right)$ (resp., $\left.\left(A_{2}, B_{2}\right)\right)$ is exactly controllable in time $2 \xi$ (resp., $2(1-\xi)$ ). The aim of this section is to describe, to some extent, the space of the states in $X^{1} \times X^{2}$ which are reachable by means of an input function $u \in L^{2}[0, T]$, with sufficiently large $T$. 
We cannot give a precise characterization of this reachable space but we give sharp embedding results in appropriate Sobolev spaces.

For $s>-\frac{1}{2}$, we introduce the space $\mathcal{W}_{s} \subset X^{1} \times X^{2}$ of quadruples of functions $\left(w_{1}^{0}, w_{1}^{1}, w_{2}^{0}, w_{2}^{1}\right)$ satisfying

$$
\begin{gathered}
\left(w_{1}^{0}, w_{1}^{1}, w_{2}^{0}, w_{2}^{1}\right) \in H^{s+1}(0, \xi) \times H^{s}(0, \xi) \times H^{s+1}(\xi, 1) \times H^{s}(\xi, 1), \\
w_{1}^{0}(0)=0, \quad w_{2}^{0}(1)=0, \quad w_{1}^{0}(\xi)=w_{2}^{0}(\xi) .
\end{gathered}
$$

Denote by $\mathbb{Q}$ the set of rational numbers. We denote by $\mathcal{S}$ the set of all numbers $\rho \in(0,1)$ such that $\rho \notin \mathbb{Q}$ and if $\left[0, a_{1}, \ldots, a_{n}, \ldots\right]$ is the expansion of $\rho$ as a continuous fraction, then $\left(a_{n}\right)$ is bounded. Note that $\mathcal{S}$ is uncountable and, by classical results on diophantine approximation (cf. [8, p. 120]), its Lebesgue measure is zero. Roughly speaking, the set $\mathcal{S}$ contains the irrationals which are "badly" approximable by rational numbers. In particular, by the Euler-Lagrange theorem (cf. [17, p. 57]) $\mathcal{S}$ contains all $\xi \in(0,1)$ such that $\xi$ is an irrational quadratic number (i.e., satisfying a second degree equation with rational coefficients). According to a classical result (see, for instance, [17]), if $\xi \in \mathcal{S}$, then there exists a constant $C_{\xi}>0$ such that

$$
\left|\xi-\frac{p}{q}\right| \geq \frac{C_{\xi}}{q^{2}} \quad \forall p, q \in \mathbb{N}
$$

We can now state our main result concerning the lack of simultaneous exact controllability of the two strings, which also gives some information on the simultaneously reachable space as a function of $\xi$.

TheOREM 5.1. Suppose that $T>\max \{4 \xi, 4(1-\xi)\}$. Then the following holds.

(a) For any $\xi \in \mathcal{S}$, all the elements of $\mathcal{W}_{0}$ can be reached in time $T$ by means of an input $u \in L^{2}[0, T]$.

(b) For almost all $\xi \in[0,1]$ and $\forall s>0$, all the states in $\mathcal{W}_{s}$ can be reached in time $T$ by means of an input $u \in L^{2}[0, T]$.

(c) The results above are sharp in the sense that, for any $\xi \in(0,1)$ and $s \in\left(-\frac{1}{2}, 0\right)$, we can find a state in $\mathcal{W}_{s}$ which is not reachable by means of an input $u \in L^{2}[0, T]$. In particular, for any $T>0$, the systems (5.1), (5.2) are not simultaneously exactly controllable in time $T$ (in the natural energy space $X^{1} \times X^{2}$ ).

As a tool in our proof, $\forall s>-\frac{1}{2}$ we introduce the space

$$
\mathcal{V}_{s}=H_{0}^{s+1}(0, \xi) \times H^{s}(0, \xi) \times H_{0}^{s+1}(\xi, 1) \times H^{s}(\xi, 1) .
$$

It is clear that $\mathcal{V}_{s}$ is a subspace of $W_{s}$ (with finite codimension). In order to prove Theorem 5.1, we notice first that for $s<\frac{1}{2}$, the reachability of $\mathcal{W}_{s}$ is equivalent to the reachability of its subspace $\mathcal{V}_{s}$. More precisely, we have the following lemma.

Lemma 5.2. Let $s \in\left(-\frac{1}{2}, \frac{1}{2}\right)$. Then all the elements of $\mathcal{W}_{s}$ can be reached in time $T$ by means of an input $u \in L^{2}[0, T]$ if and only if the same property holds for $\mathcal{V}_{s}$.

Proof. One of the implications is trivial. Take $\left(w_{1}^{0}, w_{1}^{1}, w_{2}^{0}, w_{2}^{1}\right) \in \mathcal{W}_{s}$ for some fixed $s \in\left(-\frac{1}{2}, \frac{1}{2}\right)$ and denote $\alpha=w_{1}^{0}(\xi)=w_{2}^{0}(\xi)$. Let $\psi_{1}(x, t), \psi_{2}(x, t)$ be the solutions of (5.1), (5.2) with $u=u_{\psi}$, where

$$
u_{\psi}(t)=\frac{\alpha}{T^{2}} t^{2} .
$$


It can be checked, arguing similarly as in the proof of Lemma 3.7, but differentiating twice, that

$$
\left(\psi_{1}, \dot{\psi}_{1}, \psi_{2}, \dot{\psi}_{2}\right) \in C\left([0, T] ; \mathcal{W}_{1}\right) .
$$

In particular, this implies that the above statement is true with $\mathcal{W}_{s}$ in place of $\mathcal{W}_{1}$. Moreover, we have

$$
\psi_{1}(0, T)=\psi_{2}(1, T)=0, \quad \psi_{1}(\xi, T)=\psi_{2}(\xi, T)=\alpha .
$$

The above equalities (together with $s<\frac{1}{2}$ ) imply that

$$
\left(w_{1}^{0}-\psi_{1}(\cdot, T), w_{1}^{1}-\dot{\psi}_{1}(\cdot, T), w_{2}^{0}-\psi_{2}(\cdot, T), w_{2}^{1}-\dot{\psi}_{2}(\cdot, T)\right) \in \mathcal{V}_{s} .
$$

Suppose now that all the elements of $\mathcal{V}_{s}$ can be reached in time $T$ by means of an input in $L^{2}[0, T]$. It follows that there exists an input $u_{\varphi} \in L^{2}[0, T]$ such that the solutions $\varphi_{1}, \varphi_{2}$ of (5.1) and (5.2) with $u=u_{\varphi}$ satisfy the conditions

$$
\begin{aligned}
& \varphi_{1}(x, T)=w_{1}^{0}(x)-\psi_{1}(x, T), \dot{\varphi}_{1}(x, T)=w_{1}^{1}(x)-\dot{\psi}_{1}(x, T), \quad \text { in } L^{2}[0, \xi], \\
& \varphi_{2}(x, T)=w_{2}^{0}(x)-\psi_{2}(x, T), \dot{\varphi}_{2}(x, T)=w_{2}^{1}(x)-\dot{\psi}_{2}(x, T), \quad \text { in } L^{2}[\xi, 1] .
\end{aligned}
$$

If we define the input $u \in L^{2}[0, T]$ by $u=u_{\psi}+u_{\varphi}$, then the corresponding solutions $w_{1}$ and $w_{2}$ of (5.1), (5.2) satisfy

$$
w_{1}(x, T)=w_{1}^{0}(x), \dot{w}_{1}(x, T)=w_{1}^{1}(x), w_{2}(x, T)=w_{2}^{0}(x), \dot{w}_{2}(x, T)=w_{2}^{1}(x) .
$$

Thus, the elements of $\mathcal{W}_{s}$ can be reached in time $T$ by an input $u \in L^{2}[0, T]$.

The main tool used in the proof of Theorem 5.1 is a recent generalization of a classical inequality of Ingham. This result was first proved in Jaffard, Tucsnak, and Zuazua [14] for $T>\frac{12 \sqrt{6}}{\delta}$ and then improved in Baiochi, Komornik, and Loreti [4] for $T>\frac{4 \pi}{\delta}$. Its statement (following [4]) is the following theorem.

THEOREM 5.3. Let $M>0$ and let $\left(\lambda_{n}\right)$ be a strictly increasing real sequence over $\mathbb{Z}$ satisfying

$$
\lambda_{n+2}-\lambda_{n} \geq \delta>0 \quad \forall n \in \mathbb{Z} \text { with }|n| \geq M
$$

Then $\forall T>\frac{4 \pi}{\delta}$ there exist constants $C_{1}, C_{2}>0$ such that

$$
\begin{gathered}
C_{1} \sum\left[\left(\left|a_{n}\right|^{2}+\left|a_{n+1}\right|^{2}\right)\left|\lambda_{n+1}-\lambda_{n}\right|^{2}+\left|a_{n}+a_{n+1}\right|^{2}\right] \leq \int_{0}^{T}\left|\sum a_{n} e^{i \lambda_{n} t}\right|^{2} d t \\
\leq C_{2} \sum\left[\left(\left|a_{n}\right|^{2}+\left|a_{n+1}\right|^{2}\right)\left|\lambda_{n+1}-\lambda_{n}\right|^{2}+\left|a_{n}+a_{n+1}\right|^{2}\right] \quad \forall\left(a_{n}\right) \in l^{2} .
\end{gathered}
$$

Let us now consider the initial and boundary value problems

$$
\ddot{\phi}_{1}(x, t)-\frac{\partial^{2} \phi_{1}}{\partial x^{2}}(x, t)=0 \quad \forall x \in(0, \xi) \quad \forall t \in(0, \infty),
$$




$$
\begin{gathered}
\phi_{1}(0, t)=\phi_{1}(\xi, t)=0 \quad \forall t \in(0, \infty), \\
\phi_{1}(x, 0)=\phi_{1}^{0}(x), \quad \dot{\phi}_{1}(x, 0)=\phi_{1}^{1}(x) \quad \forall x \in(0, \xi),
\end{gathered}
$$

and

$$
\begin{gathered}
\ddot{\phi}_{2}(x, t)-\frac{\partial^{2} \phi_{2}}{\partial x^{2}}(x, t)=0 \quad \forall x \in(\xi, 1) \quad \forall t \in(0, \infty), \\
\phi_{2}(1, t)=\phi_{2}(\xi, t)=0 \quad \forall t \in(0, \infty), \\
\phi_{2}(x, 0)=\phi_{2}^{0}(x), \quad \dot{\phi}_{2}(x, 0)=\phi_{2}^{1}(x) \quad \forall x \in(\xi, 1) .
\end{gathered}
$$

We will use the following duality result, which is related to Proposition 3.2. This result follows from Theorem 2.1 in Dolecki and Russell [9] or from the HUM method of Lions (see [20]).

LEMMA 5.4. The space of the states of (5.1), (5.2) which can be reached by means of the same input $u \in L^{2}[0, T]$ contains the space $\mathcal{V}_{s}, s \in\left(-\frac{1}{2}, \frac{1}{2}\right)$ if and only if there exist $C, T>0$ such that the solutions $\phi_{1}, \phi_{2}$ of (5.7)-(5.12) satisfy

$$
\begin{aligned}
\int_{0}^{T}\left|\frac{\partial \phi_{2}}{\partial x}(\xi, t)-\frac{\partial \phi_{1}}{\partial x}(\xi, t)\right|^{2} d t \\
\quad \geq\left(\left\|\phi_{1}^{0}\right\|_{H^{-s}(0, \xi)}^{2}+\left\|\phi_{1}^{1}\right\|_{H^{-1-s}(0, \xi)}^{2}+\left\|\phi_{2}^{0}\right\|_{H^{-s}(\xi, 1)}^{2}+\left\|\phi_{2}^{1}\right\|_{H^{-1-s}(0, \xi)}^{2}\right)
\end{aligned}
$$

$\forall\left(\phi_{1}^{0}, \phi_{1}^{1}, \phi_{2}^{0}, \phi_{2}^{1}\right) \in\left(H^{2}(0, \xi) \cap H_{0}^{1}(0, \xi)\right) \times H_{0}^{1}(0, \xi) \times\left(H^{2}(\xi, 1) \cap H_{0}^{1}(\xi, 1)\right) \times H_{0}^{1}(\xi, 1)$.

Proof of Theorem 5.1. If $\phi_{1}^{0} \in H^{2}(0, \xi) \cap H_{0}^{1}(0, \xi), \phi_{1}^{1} \in H_{0}^{1}(0, \xi), \phi_{2}^{0} \in H^{2}(\xi, 1) \cap$ $H_{0}^{1}(\xi, 1), \phi_{2}^{1} \in H_{0}^{1}(\xi, 1)$, it is known that we have the expansions

$$
\begin{aligned}
& \left.\begin{array}{l}
\phi_{1}^{0}(x)=\quad \sum_{n \geq 1} c_{n} \sin \left(\frac{n \pi x}{\xi}\right) \\
\phi_{1}^{1}(x)=\frac{\pi}{\xi} \sum_{n \geq 1} n d_{n} \sin \left(\frac{n \pi x}{\xi}\right)
\end{array}\right\} x \in(0, \xi), \\
& \left.\begin{array}{l}
\phi_{2}^{0}(x)=\quad \sum_{n \geq 1} e_{n} \sin \left(\frac{n \pi(1-x)}{1-\xi}\right) \\
\phi_{2}^{1}(x)= \\
\frac{\pi}{1-\xi} \sum_{n \geq 1} n f_{n} \sin \left(\frac{n \pi(1-x)}{1-\xi}\right)
\end{array}\right\} x \in(\xi, 1),
\end{aligned}
$$

where the sequences $\left(n^{2} c_{n}\right),\left(n^{2} d_{n}\right),\left(n^{2} e_{n}\right)$, and $\left(n^{2} f_{n}\right)$ are in $l^{2}$. A standard calculation shows that the solutions $\phi_{1}, \phi_{2}$ of (5.7)-(5.12) are given by

$$
\begin{gathered}
\phi_{1}(x, t)=\sum_{n \in \mathbb{Z}} a_{n} e^{i \frac{n \pi}{\xi} t} \sin \left(\frac{n \pi x}{\xi}\right), \quad x \in(0, \xi), \\
\phi_{2}(x, t)=\sum_{n \in \mathbb{Z}} b_{n} e^{i \frac{n \pi}{1-\xi} t} \sin \left(\frac{n \pi(1-x)}{1-\xi}\right), \quad x \in(\xi, 1),
\end{gathered}
$$


where

$$
\begin{gathered}
a_{n}= \begin{cases}\frac{c_{n}-i d_{n}}{2} & \text { for } n \geq 1, \\
\frac{c_{-n}+i d_{-n}}{2} & \text { for } n \leq-1, \\
0 & \text { for } n=0,\end{cases} \\
b_{n}= \begin{cases}\frac{e_{n}-i f_{n}}{2} & \text { for } n \geq 1, \\
\frac{e_{-n}+i f_{-n}}{2} & \text { for } n \leq-1, \\
0 & \text { for } n=0 .\end{cases}
\end{gathered}
$$

If we denote by $\left(\lambda_{n}\right)_{n \in \mathbb{Z}}$ the strictly increasing sequence formed by the elements of the set

$$
\Lambda=\left[\cup_{n \in \mathbb{Z}}\left\{\frac{n \pi}{\xi}\right\}\right] \bigcup\left[\cup_{n \in \mathbb{Z}}\left\{\frac{n \pi}{1-\xi}\right\}\right]
$$

we can easily check that

$$
\lambda_{n+2}-\lambda_{n} \geq \operatorname{in}\left\{\frac{\pi}{\xi}, \frac{\pi}{1-\xi}\right\} \quad \forall n \in \mathbb{Z} .
$$

On the other hand, from (5.3) it easily follows (see [13] for details) that, $\forall \xi \in \mathcal{S}$, there exists a constant $C_{\xi}>0$ with

$$
\lambda_{n+1}-\lambda_{n} \geq \frac{C_{\xi}}{\left|\lambda_{n}\right|} \quad \forall n \in \mathbb{Z}^{*},
$$

where $\mathbb{Z}^{*}=\mathbb{Z} \backslash\{0\}$. Moreover (5.13), (5.14) imply

$$
\frac{\partial \phi_{2}}{\partial x}(\xi, t)-\frac{\partial \phi_{1}}{\partial x}(\xi, t)=\sum_{n \in \mathbb{Z}^{*}}(-1)^{n+1} n \pi\left(\frac{a_{n}}{\xi} e^{i \frac{n \pi t}{\xi}}+\frac{b_{n}}{1-\xi} e^{i \frac{n \pi t}{1-\xi}}\right),
$$

which yields

$$
\frac{\partial \phi_{2}}{\partial x}(\xi, t)-\frac{\partial \phi_{1}}{\partial x}(\xi, t)=\sum_{n \in \mathbb{Z}^{*}} k_{n} \lambda_{n} e^{i \lambda_{n} t}
$$

with the sequence $\left(k_{n}\right)$ satisfying

$$
\sum_{n \in \mathbb{Z}^{*}}\left|k_{n}\right|^{2}=\sum_{n \in \mathbb{Z}^{*}}\left(\left|a_{n}\right|^{2}+\left|b_{n}\right|^{2}\right) .
$$

Relations (5.18), (5.20), (5.21), and Theorem 5.3 imply that there exists a constant $K_{\xi}>0$ such that

$$
\int_{0}^{T}\left|\frac{\partial \phi_{2}}{\partial x}(\xi, t)-\frac{\partial \phi_{1}}{\partial x}(\xi, t)\right|^{2} d t \geq K_{\xi} \sum_{n \in \mathbb{Z}}\left(\left|a_{n}\right|^{2}+\left|b_{n}\right|^{2}\right)
$$

$\forall \xi \in \mathcal{S}$ and $\forall T>\max \{4 \xi, 4(1-\xi)\}$. Inequality (5.22) combined with Lemma 5.4 implies that the elements in $\mathcal{V}_{0}$ are reachable by means of an input in $L^{2}[0, T]$. By using Lemma 5.2 we obtain assertion (a) of Theorem 5.1. 
According to Lemma 7.3 in [13], $\forall \varepsilon>0$ there exists a set $B_{\varepsilon} \subset(0,1)$, of Lebesgue measure 1 , such that $\forall \xi \in B_{\varepsilon}$, there exists a constant $C_{\xi}>0$ with

$$
\lambda_{n+1}-\lambda_{n} \geq \frac{C_{\xi}}{\left|\lambda_{n}\right|^{1+\varepsilon}} \quad \forall n \in \mathbb{Z}^{*}
$$

Relations (5.20), (5.21), (5.23), and Theorem 5.3 imply that there exists a constant $K_{\xi}>0$ such that

$$
\int_{0}^{T}\left|\frac{\partial \phi_{2}}{\partial x}(\xi, t)-\frac{\partial \phi_{1}}{\partial x}(\xi, t)\right|^{2} d t \geq K_{\xi} \sum_{n \in \mathbb{Z}}\left(\frac{\left|a_{n}\right|^{2}+\left|b_{n}\right|^{2}}{\left|\lambda_{n}\right|^{2 \varepsilon}}\right)
$$

$\forall \xi \in B_{\varepsilon}$ and $\forall T>\max \{4 \xi, 4(1-\xi)\}$. Lemma 5.4 combined with (5.24) implies that $\forall s \in\left(0, \frac{1}{2}\right)$, the elements in $\mathcal{V}_{s}$ are reachable by an input in $L^{2}[0, T]$. By applying again Lemma 5.2 we get assertion (b) of Theorem 5.1 for $s<\frac{1}{2}$. For $s \geq \frac{1}{2}$ the assertion remains true because $\mathcal{W}_{s} \subset \mathcal{W}_{r}$ for $s>r$.

In order to prove assertion (c) we notice that, $\forall \xi \in(0,1)$, we can use the continuous fractions expansion of $\frac{1-\xi}{\xi}$ to construct a sequence $(p(n))$ with values in $\mathbb{N}$, with $\lim _{n \rightarrow \infty} p(n)=\infty$, such that

$$
\lambda_{p(n)+1}-\lambda_{p(n)} \leq \frac{C}{p(n)} \quad \forall n \in \mathbb{N}
$$

If we denote by $\left(\phi_{1 n}\right)$ (resp., by $\left.\left(\phi_{2 n}\right)\right)$ the sequence of solutions of (5.7)-(5.9) (resp., of $(5.10)-(5.12))$ having initial data $\left(\sin \left(\frac{p(n) \pi}{\xi}\right), 0\right)$ (resp., $\left(\sin \left(\frac{(p(n)+1) \pi}{1-\xi}\right), 0\right)$, relations (5.13), (5.14), and (5.25) imply that

$$
\lim _{n \rightarrow \infty} \frac{\int_{0}^{T}\left|\frac{\partial \phi_{2 n}}{\partial x}(\xi, t)-\frac{\partial \phi_{1 n}}{\partial x}(\xi, t)\right|^{2} d t}{\left\|\phi_{1 n}(0)\right\|_{H^{s}(0, \xi)}^{2}+\left\|\phi_{2 n}(0)\right\|_{H^{s}(\xi, 1)}^{2}}=0
$$

$\forall s<0$. Using again Lemma 5.4 we conclude that (c) also holds.

Remark 5.5. The fact that (5.24) holds for any $T>\max \{4 \xi, 4(1-\xi)\}$ was proved in [4]. Earlier versions of this inequality (corresponding to larger values of $T$ ) were given in [13] and [14]. Notice that (5.24) and the standard duality argument imply only reachability of elements in $\mathcal{V}_{s}$. In order to get the reachability of elements in $\mathcal{W}_{s}$ we need a different argument, namely Lemma 5.2.

Remark 5.6. Intuitively it does not seem reasonable to have a minimal simultaneous reachability time depending on $\xi$. This question and other related issues (simultaneous approximate controllability, simultaneous spectral controllability) are tackled in [3]. In this work it is shown that the minimal time for these various types of controllability is $T=2$.

Acknowledgments. The authors wish to thank Enrique Zuazua for suggesting a method to obtain the optimal approximate controllability time in Lemma 3.4. We are grateful to Sergei Avdonin, Francis Conrad, Scott Hansen, and Olof Staffans for helpful discussions and references. 


\section{REFERENCES}

[1] G. Avalos And G. Weiss, The Wave Equation as a Conservative Regular Linear System, in preparation.

[2] S.-A. Avdonin and S.A. Ivanov, Families of Exponentials-The Method of Moments in Controllability Problems for Distributed Parameter Systems, Cambridge University Press, Cambridge, UK, 1995.

[3] S.-A. Avdonin and M. Tucsnak, Simultaneous Controllability in Short Time for Two Elastic Strings, preprint, 1999.

[4] C. Baiochi, V. Komornik, And P. Loreti, Ingham type theorems and applications to control theory, Boll. Un. Mat. Ital. B, II B (1999), pp. 33-63.

[5] C. Bardos, G. Lebeau, And J. Rauch, Sharp sufficient conditions for the observation, control and stabilization of waves from the boundary, SIAM J. Control Optim., 30 (1992), pp. 10241065.

[6] H. Brezis, Functional Analysis, Springer-Verlag, New York, 1988 (French original published by Masson, Paris).

[7] P.L. Butzer and H. Berens, Semi-groups of Operators and Approximation, Springer-Verlag, Berlin, 1967.

[8] J.W.S. CAssels, An Introduction to Diophantine Approximation, Cambridge University Press, Cambridge, UK, 1965.

[9] S. Dolecki AND D.L. Russell, A general theory of observation and control, SIAM J. Control Optim., 15 (1977), pp. 185-220.

[10] S.W. HANSEN, Boundary control of a one-dimensional linear thermoelastic rod, SIAM J. Control Optim., 32 (1994), pp. 1052-1074.

[11] S.W. Hansen and G. Weiss, New results on the operator Carleson measure criterion, IMA J. Math. Control Inform., 14 (1997), pp. 3-32.

[12] S.W. HANSEn And B.Y. Zhang, Boundary control of a linear thermoelastic beam, J. Math. Anal. Appl., 210 (1997), pp. 182-205.

[13] S. Jaffard, M. Tucsnak, AND E. ZuazuA, Singular internal stabilization of the wave equation, J. Differential Equations, 145 (1998), pp. 184-215.

[14] S. Jaffard, M. Tucsnak, And E. Zuazua, On a theorem of Ingham, J. Fourier Anal. Appl., 3 (1997), pp. 577-582.

[15] A. Haraux, Remarques sur la contrôlabilité ponctuelle et spectrale de systèmes distribuées, Publications du laboratoire d'analyse numérique R89017, Preprint 2000/4, Institut Elie Cartan de Nancy, France, 1989.

[16] J.E. Lagnese and J.L. Lions, Modelling, Analysis and Control of Thin Plates, Masson, Paris, 1988.

[17] S. LANG, Introduction to Diophantine Approximations, Addison-Wesley, New York, 1966.

[18] I. Lasiecka And R. TRiggiani, A cosine operator approach to the modelling of $L_{2}\left(0, t ; L^{2}(\Gamma)\right)$ boundary input hyperbolic equations, Appl. Math. Optim., 7 (1981), pp. 35-83.

[19] I. Lasiecka and R. TRiggiani, Regularity of hyperbolic equations under boundary terms, Appl. Math. Optim., 7 (1981), pp. 35-83.

[20] J.-L. Lions, Controlabilité exacte perturbations et stabilisation de systèmes distribués 1, Rech. Math. Appl. 8, Masson, Paris, 1988.

[21] V. Komornik, Exact Controllability and Stabilization-The Multiplier Method, RAM Res. Appl. Math., John Wiley, Chichester, UK, Masson, Paris, 1994.

[22] D.L. Russell, The Dirichlet-Neumann boundary control problem associated with Maxwell's equations in a cylindrical region, SIAM J. Control Optim., 24 (1986), pp. 199-229.

[23] D.L. Russell and G. Weiss, A general necessary condition for exact observability, SIAM J. Control Optim., 32 (1994), pp. 1-23.

[24] G. WeIss, Admissibility of unbounded control operators, SIAM J. Control Optim., 27 (1989), pp. $527-545$.

[25] G. WeISs, Admissible observation operators for linear semigroups, Israel J. Math., 65 (1989), pp. $17-43$.

[26] G. WeIss, Regular linear systems with feedback, Math. Control Signals Systems, 7 (1994), pp. 23-57.

[27] E. Zuazua, An Introduction to Exact Controllability for Distributed Systems, Lecture notes, University of Lisbon, Lisbon, Portugal, 1990. 